\title{
Exact Theory of the (Einstein) Gravitational Field in an Arbitrary Background Space-Time
}

\author{
L. P. Grishchuk ${ }^{1}$, A. N. Petrov ${ }^{1}$, and A. D. Popova ${ }^{2}$
}

1 Sternberg Astronomical Institute, SU-119899 Moscow, USSR

2 Institute of Scientific Information, SU-125219 Moscow, USSR

\begin{abstract}
The Lagrangian based theory of the gravitational field and its sources at the arbitrary background space-time is developed. The equations of motion and the energy-momentum tensor of the gravitational field are derived by applying the variational principle. The gauge symmetries of the theory and the associated conservation laws are investigated. Some properties of the energymomentum tensor of the gravitational field are described in detail and the examples of its application are given. The desire to have the total energymomentum tensor as a source for the linear part of the gravitational field leads to the universal coupling of gravity with other fields (as well as to the selfinteraction) and finally to the Einstein theory.
\end{abstract}

\section{Introduction}

Investigations on general relativity (GR) are frequently being carried out under the assumption that there exists some background space-time. Problems of postNewtonian equations of motion, generation and propagation of weak gravitational waves, quantization of weak gravitational field apply the notion of flat background space-time [1-3]. A curved background space-time (especially, cosmological and black hole geometries) can be used when the propagation and amplification of perturbations or vacuum polarization and particle creation effects are considered. The "background field method" is developed for treating the various quantum fields at the classical background [4]. The notion of background space-time, primarily flat, is also invoked when interpreting the solutions of GR equations.

Investigations of this type are being carried out, as a rule, in the linear approximation, without taking into account the "back reaction" of perturbations, or, in a better case, by successive approximations. Many additional constructions are used, like averaging over space-time volumes, specific choice of coordinate conditions, asymptotically Cartesian coordinates, etc. Unfortunately, it is not 
always clear which results, obtained in this way, have general meaning and which depend on additional constructions.

In our opinion, this approach deserves further study and more thorough and compact formulation. Among other things it sheds some light on the problem of energy and momentum of the gravitational field. It is known that the standard (pseudotensor) description is full of ambiguities. Nevertheless, an unambiguous answer to the question of which quantity plays the role of the energy-momentum tensor $t_{\mu \nu}$ of the gravitational field is needed, for example, when computing the conformal anomaly for the trace of $t_{\mu \nu}$, similar to what was done for other physical fields in a curved background space-time. (We will consider this problem in a different publication.)

There are well known papers (see, for example, [5-8]) where GR is treated as a theory of a nonlinear tensor field at a flat background space-time. These works paid much attention to the issue of how natural and unavoidable the Einstein equations are; however the actual content of this approach has been developed to a lesser extent.

In the present paper, following in many respects [8] and [4], we give the Lagrangian formulation of the theory of the gravitational field and its sources at an arbitrary background space-time. The background (absolute) and dynamical variables are introduced from the very beginning. By using the variational principle, the field equations are derived together with the energy-momentum tensor $t_{\mu \nu}$ of the gravitational field and of other dynamical fields. The gauge symmetries of the action and of the field equations are examined, the conservation laws are discussed. Some properties of $t_{\mu \nu}$ are considered and some examples of its application are given. The theory is developed in a self-contained way and its formal equivalence to GR is established at the very end, by making some appropriate identifications.

The following notations are used. The background space-time (with signature $+,-,-,-)$ has the metric tensor $\gamma_{\mu \nu}$ and the Christoffel symbols $C_{\beta \gamma}^{\alpha}$. The background curvature tensor is $\stackrel{R}{\beta \gamma \delta}^{\alpha} \equiv \frac{\partial C_{\beta \delta}^{\alpha}}{\partial x^{\gamma}}-\ldots$, the Ricci tensor is $\stackrel{R}{\alpha \beta}_{\alpha \beta} \equiv \dot{R}_{\alpha \sigma \beta}^{\sigma}$. The ordinary derivative is denoted by a comma, and the covariant derivative - by a semicolon. No special restrictions on the coordinate system are imposed. In particular, if the space-time is flat, $R_{\beta \gamma \delta}^{\alpha}=0$, the metric tensor $\gamma_{\mu \nu}$ is not necessarily assumed to be in the Minkowski form $\eta_{\mu v}$. The functional derivatives have the usual form:

$$
\frac{\delta L\left(\varphi ; \varphi_{, \alpha} ; \varphi_{, \alpha \beta}\right)}{\delta \varphi}=\frac{\partial L}{\partial \varphi}-\left(\frac{\partial L}{\partial \varphi_{, \alpha}}\right)_{, \alpha}+\left(\frac{\partial L}{\partial \varphi_{, \alpha, \beta}}\right)_{, \alpha \beta} .
$$

The symmetric energy-momentum tensor for the arbitrary action

$$
S=\frac{1}{c} \int d^{4} x L
$$

is defined as in [2]:

$$
T_{\mu \nu} \equiv \frac{2}{\sqrt{-\gamma}} \frac{\delta L}{\delta \gamma^{\mu \nu}} \equiv 2 \frac{\delta L}{\delta\left(\sqrt{-\gamma} \gamma^{\mu \nu}\right)}-\gamma_{\mu \nu} \gamma^{\alpha \beta} \frac{\delta L}{\delta\left(\sqrt{-\gamma} \gamma^{\alpha \beta}\right)},
$$




\section{The Flat and the Ricci-Flat Background Space-Time}

Obviously, the case of the flat background space-time, $\hat{R}_{\beta \gamma \delta}^{\alpha}=0$, is privileged since, in that case, GR recalls the theories of other physical fields to the largest extent. However, most of the relations presented below are valid also in the more general case: $\dot{R}_{\beta \gamma \delta}^{\alpha} \neq 0, \hat{R}_{\alpha \beta}=0$, so we stick to the Ricci-flat backgrounds. Differences arise only when treating the integral conservation laws, and they will be discussed later (Sect. 4).

\section{2a) Gravitational Field Without Matter Sources}

Let the tensor fields $h^{\mu v}$ and $K_{\cdot \mu \nu}^{\alpha}$ be given in a Ricci-flat background:

$$
R_{\alpha \beta}^{\circ}=0 \text {. }
$$

The tensor $h^{\mu \nu}$ is symmetric and the tensor $K_{\cdot \mu \nu}^{\alpha}$ is symmetric with respect to the lower (last) two indices. Following [8], we apply the first order formalism and consider $h^{\mu \nu}$ and $K_{\cdot{ }_{\mu \nu}}^{\alpha}$ as independent variables. Take the action for the gravitational field in the form:

$$
S=-\frac{1}{2 c \kappa} \int d^{4} x L^{g}
$$

where

$$
L^{g} \equiv \sqrt{-\gamma} h^{\mu \nu}\left(K_{\cdot \mu \nu ; \alpha}^{\alpha}-K_{\mu ; v}\right)+\sqrt{-\gamma}\left(\gamma^{\mu \nu}+h^{\mu \nu}\right)\left(K_{\cdot \mu \nu}^{\alpha} K_{\alpha}-K_{\cdot{ }_{\mu \beta}}^{\alpha} K_{\cdot v \alpha}^{\beta}\right) .
$$

Here and below we use the notations:

$$
K_{\alpha} \equiv K_{\sigma \alpha}^{\sigma}, \quad C_{\alpha} \equiv C_{\sigma \alpha}^{\sigma} ; \quad \tilde{h}^{\mu \nu} \equiv \sqrt{-\gamma} h^{\mu \nu}, \quad \tilde{\gamma}^{\mu \nu} \equiv \sqrt{-\gamma} \gamma^{\mu \nu} .
$$

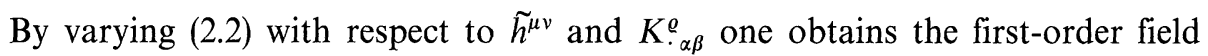
equations:

$$
\begin{aligned}
r_{\mu \nu}(K) & \equiv \frac{\delta L^{g}}{\delta \tilde{h}^{\mu \nu}} \equiv K_{\mu \nu ; \alpha}^{\alpha}-\frac{1}{2} K_{\mu ; \nu}-\frac{1}{2} K_{v ; \mu}+K_{\mu \nu}^{\alpha} K_{\alpha}-K^{\alpha}{ }_{\mu \beta} K^{\beta}{ }_{v \alpha}=0, \\
f_{\varrho}^{\alpha \beta}(K, h) & \equiv \frac{\delta L^{g}}{\delta K_{\cdot \mu \nu}^{\tau}}\left(\delta_{\varrho}^{\tau} \delta_{\mu}^{\alpha} \delta_{v}^{\beta}-\frac{1}{3} \delta_{v}^{\tau} \delta_{\mu}^{\alpha} \delta_{\varrho}^{\beta}-\frac{1}{3} \delta_{v}^{\tau} \delta_{\mu}^{\beta} \delta_{\varrho}^{\alpha}\right) \\
& \equiv-\tilde{h}_{; \varrho}^{\alpha \beta}+\left(\tilde{\gamma}^{\alpha \beta}+\tilde{h}^{\alpha \beta}\right) K_{\varrho}-\left(\tilde{\gamma}^{\alpha \mu}+\tilde{h}^{\alpha \mu}\right) K^{\beta}{ }_{\mu \varrho}-\left(\tilde{\gamma}^{\beta \mu}+\tilde{h}^{\beta \mu}\right) K_{\mu \varrho}^{\alpha}=0 .
\end{aligned}
$$

From (2.4), (2.5) one can obtain the second order equations. For this one has to take the covariant derivative with respect to $\tau$ from the equation

$$
\frac{1}{2 \sqrt{-\gamma}}\left(f_{\mu \nu}^{\cdots \cdot \tau}+f_{\nu \mu}^{\cdots \cdot \tau}-f_{\mu \nu}^{\tau \cdot \cdot}+\frac{1}{2} \gamma_{\mu \nu} f_{\alpha}^{\tau \alpha \cdot}\right)=0
$$

and use (2.4). Then one obtains:

$$
G_{\mu \nu}^{L}(h)=-(K K)_{\mu \nu}+\frac{1}{2} \gamma_{\mu \nu}(K K)_{\cdot \alpha}^{\alpha}+Q_{\mu \nu},
$$


where

$$
\begin{aligned}
& 2 G^{L}{ }_{\mu \nu}(h) \equiv h_{\mu \nu}^{; \alpha} ; \alpha+\gamma_{\mu v} h_{; \alpha ; \beta}^{\alpha \beta}-h_{v ; \mu ; \alpha}^{\alpha}-h_{\mu ; v ; \alpha}^{\alpha}, \\
& (K K)_{\mu \nu} \equiv K_{\mu \nu}^{\alpha} K_{\alpha}-K^{\alpha}{ }_{\mu \beta} K^{\beta}{ }_{v \alpha} \text {, } \\
& Q_{\mu \nu} \equiv Q_{\mu v ; \tau}^{\tau}, \\
& 2 Q^{\tau}{ }_{\mu \nu} \equiv-\gamma_{\mu \nu}{ }^{\alpha \beta} K^{\tau}{ }_{\alpha \beta}+h_{\mu \nu} K^{\tau}-h_{\mu}{ }^{\tau} K_{v}-h_{v}{ }^{\tau} K_{\mu}+h^{\beta \tau}\left(K^{\alpha}{ }_{\mu \beta} \gamma_{\alpha \nu}+K^{\alpha}{ }_{\nu \beta} \gamma_{\alpha \mu}\right) \\
& +h_{\mu}^{\beta}\left(K_{\nu \beta}^{\tau}-K_{\beta e}^{\alpha} \gamma^{\gamma^{e \tau}} \gamma_{\alpha \nu}\right)+h_{v}^{\beta}\left(K_{\mu \beta}^{\tau}-K^{\alpha}{ }_{\alpha \beta} \gamma^{\rho \tau} \gamma_{\alpha \mu}\right) \text {. }
\end{aligned}
$$

The right-hand side of (2.6) is identically equal to $-\frac{1}{\sqrt{-\gamma}} \frac{\delta L^{g}}{\delta \gamma^{\mu \nu}}$. If one uses the definition (1.1) then the field equations (2.6) can be rewirtten in the form:

$$
G_{\mu \nu}^{L}(h)=\kappa t_{\mu v}(h, K),
$$

where $t_{\mu \nu}$ is the symmetric energy-momentum tensor of the gravitational field:

$$
\kappa t_{\mu \nu} \equiv-\frac{1}{\sqrt{-\gamma}} \frac{\delta I^{g}}{\delta \gamma^{\mu \nu}} \equiv-(K K)_{\mu \nu}+\frac{1}{2} \gamma_{\mu \nu}(K K)_{\cdot \alpha}^{\alpha}+Q_{\mu \nu} .
$$

(Recall that the notion of the energy-momentum tensor of the gravitational field has been already present in the early works [9].)

Equation (2.8) could be also derived without using the first order formalism. For this one has to consider $K^{\alpha}{ }_{\mu \nu}$ as the known functions of $h^{\mu v}$ and $h^{\mu \nu}{ }_{, \alpha}$, defined by Eq. (2.5). By varying $L^{g}$ with respect to $\widetilde{h^{\mu \nu}}$ one obtains Eq. (2.8) where $K^{\alpha}{ }_{\mu v}$ have to be understood as the specific expressions, following from (2.5).

Note that the identity $G_{\mu \nu}^{L ; \nu} \equiv 0$ is satisfied due to Eq. (2.1):

$$
2 G_{\mu \nu}^{L ; v} \equiv \stackrel{\circ}{\lambda v ; \mu}^{\lambda \nu}-2\left(R_{\mu \lambda}^{\circ} h^{\lambda v}\right)_{; v} .
$$

As a consequence of the field equations one has $t_{\mu \nu}{ }^{;}=0$.

Equation (2.8) has the obvious interpretation of the field equations for the field, possessing the self-interaction. The left hand side (1.h.s.) of (2.8) is the covariant generalization of the field operator for the massless, spin 2, tensor field. The r.h.s. of (2.8) is the energy-momentum tensor of the same field.

The expression for $t_{\mu \nu}$, after the exclusion of $K^{\alpha}{ }_{\mu \nu}$, contains the second derivatives of $h^{\mu v}$ in the form of terms like $h^{\alpha \beta} h_{\mu v ; \alpha ; \beta}$. Sometimes such a property is regarded as a disadvantage of a theory. However, in the present case, this property is very natural. If the aforementioned terms were absent, the propagation of the gravitational field would be determined by the differential operator $G_{\mu v}^{L}$. In particular, at the flat background, the characteristics of Eq. (2.8) would be the straight null geodesics of the Minkowski space-time. Then, by using the propagating graviational field one would be able to observe the flat geometry of the world even in the presence of a gravitational field. Such a conclusion would be in a severe conflict with the whole spirit of GR.

The equivalence of the equations of the developed theory with the vacuum Einstein equations (without sources) is revealed by the identifications

$$
\tilde{\gamma}^{\mu \nu}+\tilde{h}^{\mu \nu} \equiv \sqrt{-g} g^{\mu \nu}, \quad C_{\mu \nu}^{\alpha}+K_{\mu \nu}^{\alpha} \equiv \Gamma_{\mu \nu}^{\alpha} .
$$


Then, Eq. (2.5) are just the usual relation between $g_{\alpha \beta}$ and $\Gamma_{\mu v}^{\alpha}$, and Eq. (2.4) [together with (2.1)] is equivalent to

$$
R_{\mu \nu} \equiv \Gamma_{\mu \nu, \alpha}^{\alpha}-\Gamma_{\mu, v}+\Gamma_{\mu \nu}^{\alpha} \Gamma_{\alpha}-\Gamma_{\mu \beta}^{\alpha} \Gamma_{v \alpha}^{\beta}=0 .
$$

The Lagrangian $L^{g}$ transforms into the Gilbert Lagrangian $\sqrt{-g} R$, if one adds to $L^{g}$ the terms

$$
L^{0} \equiv \sqrt{-\gamma} R^{\circ}, \quad L^{1} \equiv \tilde{h}^{\mu \nu} R_{\mu \nu}^{\circ}, \quad L^{1 \prime} \equiv\left(\tilde{\gamma}^{\mu \nu} K^{\alpha}{ }_{\mu \nu}-\tilde{\gamma}^{\alpha \varrho} K_{\varrho}\right)_{, \alpha} .
$$

These terms, after varying them by $\tilde{h}^{\mu \nu}$ and $K^{\alpha}{ }_{\mu \nu}$, do not change the field equations (2.4), (2.5). The contribution from $L^{1}$ vanishes due to Eq. (2.1).

This approach can be extended to the theory with the cosmological $\Lambda$-term. Equation (2.1) will be replaced by $\dot{R}_{\alpha \beta}-\Lambda \gamma_{\alpha \beta}=0$ and the 1.h.s. of Eq. (2.6) will be replaced by $G_{\mu \nu}^{L}+\Lambda h_{\mu \nu}$. The covariant divergence from this expression is still equal to zero identically.

Let us discuss briefly the question of the "back reaction" of $t_{\mu \nu}$ on the background. Here, two points of view are possible. According to the first, the background metric $\gamma_{\mu \nu}$ remains fixed regardless of the accuracy with which Eq. (2.8) are solved. From this point of view, to reckon $t_{\mu \nu}$ means just to take into account the nonlinearities in the equation for $h^{\mu \nu}$. According to the second point of view, one or another part of the nonlinear solution for $h^{\mu \nu}$ must be added to $\gamma^{\mu \nu}$ which leads to the change of the background metric. The extreme realization of this procedure is a complete merging of $\gamma^{\mu \nu}$ and $h^{\mu \nu}$ according to (2.11). These considerations on the "back reaction" apply, also to the other variants of the theory, considered below.

\section{2b) Gravitational Field with Matter Sources}

Introduce the matter fields interacting with the gravitational field. The nature of the fields and their transformation properties are not important at the moment. Denote the fields by $\varphi_{A}$, where $A$ is some general index.

Let the Lagrangian density $L^{m}$ of the matter fields including their interaction with gravity to have the form

$$
L^{m}=L^{m}\left(\gamma^{\mu v} ; \gamma_{, \alpha}^{\mu \nu} ; h^{\mu v} ; h^{\mu \nu}{ }_{\alpha} ; \varphi_{A} ; \varphi_{A, \alpha}\right),
$$

so that the total action is

$$
S=-\frac{1}{2 c \kappa} \int d^{4} x L^{g}+\frac{1}{c} \int d^{4} x L^{m} .
$$

Deriving the field equations, one obtains Eq. (2.5) again and

$$
r_{\mu \nu}(K)=2 \kappa \frac{\delta L^{m}}{\delta \tilde{h}^{\mu \nu}}
$$

instead of (2.4). By varying $S$ with respect to $\varphi_{A}$, one obtains the equations of motion for matter fields

$$
\frac{\delta L^{m}}{\delta \varphi_{A}}=0 .
$$


By the procedure similar to that described in Sect. $2 \mathrm{a}$ one can derive from Eqs. (2.14), (2.5) the equation

$$
G_{\mu \nu}^{L}(h)=\kappa\left(t_{\mu \nu}+2 \frac{\delta L^{m}}{\delta \tilde{h}^{\mu \nu}}-\gamma_{\mu \nu} \gamma^{\alpha \beta} \frac{\delta L^{m}}{\delta \tilde{h}^{\alpha \beta}}\right)
$$

The total energy-momentum tensor of the action (2.13) is, by definition,

$$
T_{\mu \nu}^{\mathrm{tot}} \equiv t_{\mu \nu}+2 \frac{\delta L^{m}}{\delta \tilde{\gamma}^{\mu \nu}}-\gamma_{\mu \nu} \gamma^{\alpha \beta} \frac{\delta L^{m}}{\delta \tilde{\gamma}^{\alpha \beta}} \equiv t_{\mu \nu}+T_{\mu \nu},
$$

where $T_{\mu \nu}$ is the energy-momentum tensor of the matter fields which includes also the contribution from their interaction with gravity. If we want the r.h.s. of (2.16) to be $\kappa T_{\mu \nu}^{\text {tot }}$, i.e. if want Eq. (2.16) to have the form

$$
G_{\mu \nu}^{L}=\kappa\left(t_{\mu \nu}+T_{\mu \nu}\right)
$$

then we must require that the following equations be satisfied:

$$
\frac{\partial L^{m}}{\partial{\widetilde{h^{\mu \nu}}}^{\mu \nu}}-\left(\frac{\partial L^{m}}{\partial \tilde{h}_{, \alpha}^{\mu \nu}}\right)_{, \alpha}=\frac{\partial L^{m}}{\partial \hat{\gamma}^{\mu \nu}}-\left(\frac{\partial L^{m}}{\partial \tilde{\gamma}^{\mu \nu}, \alpha}\right)_{, \alpha} .
$$

The sufficient condition for their fulfilment is that $L^{m}$ depends on $\tilde{\gamma}^{\mu \nu}+\widetilde{h}^{\mu v}$, i.e.

$$
L^{m}=L^{m}\left[\tilde{\gamma}^{\mu \nu}+\tilde{h}^{\mu \nu} ;\left(\tilde{\gamma}^{\mu \nu}+{\widetilde{h^{\mu \nu}}}_{, \alpha} ; \varphi_{A} ; \varphi_{A, \alpha}\right] .\right.
$$

This condition symbolizes the universal coupling of gravitational fields with other physical fields. The theory based on the action (2.13) and supplied with the condition (2.19) is equivalent to GR. To show this one has to make the identifications (2.11) and use the equality

$$
\frac{\delta L^{m}}{\delta \tilde{\gamma}^{\mu \nu}}=\frac{\delta L^{m}}{\delta\left(\sqrt{-g} g^{\mu \nu}\right)},
$$

which is valid because of (2.19).

A consequence of (2.18) is a covariant conservation law

$$
\left(t^{\mu \nu}+T^{\mu \nu}\right)_{; \nu}=0 .
$$

It is interesting to note that the desire to have $T_{\mu \nu}^{\text {tot }}$ as a source in Eq. (2.16) restricts the functional dependence of $L^{m}$ even if $L^{m}$ is written down in a more general form than (2.12). For instance, if $L^{m}$ contains $K^{\alpha}{ }_{\mu \nu}$ explicitly, then the condition analogous to (2.19) will take the form

$$
L^{m}=L^{m}\left[\tilde{\gamma}^{\mu \nu}+\tilde{h}^{\mu \nu} ;\left(\tilde{\gamma}^{\mu \nu}+\tilde{h}^{\mu \nu}\right)_{, \alpha} ; C_{\mu \nu}^{\alpha}+K_{\mu \nu}^{\alpha} ; \varphi_{A} ; \varphi_{A, \alpha}\right] .
$$

Once again one returns to the requirement of the universal coupling of the gravitational field to other fields (compare with [10]).

It is worth recalling that a theory without the term $t_{\mu \nu}$ in the field equations (2.18) would be inconsistent. To remedy this inconsistency one either has to include the selfinteraction (the term $t_{\mu v}$ ) or has to take a nonlocal projection of $T_{\mu \nu}$ as the r.h.s. of Eq. (2.18) [11]. In the second case one obtains a curious non-Einstein theory which agrees with the presently known experimental facts, but predicts, 
among other things, that the gravitational waves, contrary to the electromagnetic ones, do not interact with the gravitational field [11]. Since this theory includes the higher derivatives, it will, likely, encounter difficulties common for such theories.

\section{A Curved (not Ricci-Flat) Background Space-Time}

\section{3a) The Background Curvature Determined by the Background Matter}

Much research, especially cosmological, deals with small perturbations of gravitational and matter fields about some basic solution satisfying the background Einstein equations,

$$
\stackrel{\circ}{R}_{\mu \nu}-\frac{1}{2} \gamma_{\mu \nu} \stackrel{R}{R}=\kappa \stackrel{\circ}{T}_{\mu \nu}\left(\stackrel{\circ}{\varphi}_{A}\right),
$$

and the background field equations for $\stackrel{\circ}{\varphi}_{A}$. It is important to develop an exact theory along these lines. Such a theory may be useful, for instance, in the circumstances where the mean values of the (quantized) gravitational field correspond to the nonvanishing curvature and Ricci tensors, i.e to a curved background space-time. The theory may help to give a detailed description of such a miraculous process as the "spontaneous birth" of the classical Universe from zero-point quantum fluctuations (see, for example [12]).

The background physical system may be described by the total Lagrangian

$$
\stackrel{\circ}{L}=-\frac{\stackrel{\circ}{L}^{g}}{2 \kappa}+\stackrel{\circ}{L}^{m}(\gamma, \stackrel{\circ}{\varphi}),
$$

where $\stackrel{\circ}{L}^{g}=\sqrt{-\gamma} R$. Equation (3.1) and the equations for $\stackrel{\circ}{\varphi}_{A}$ follow from the variational principle:

$$
\begin{gathered}
\frac{1}{\sqrt{-\gamma}}\left(\frac{\delta \stackrel{\circ}{L}^{g}}{\delta \gamma^{\mu \nu}}-2 \kappa \frac{\delta \stackrel{\circ}{L}^{m}}{\delta \gamma^{\mu \nu}}\right)=0, \\
\frac{\delta \check{L}^{\circ}}{\delta \stackrel{\circ}{\varphi}_{A}}=0 .
\end{gathered}
$$

Now, let us introduce the dynamical variables $h^{\mu v}$ and $K^{\alpha}{ }_{\mu \nu}$ for the gravitational field and $\varphi_{A}$ for matter fields. Take the Lagrangian for dynamical fields in the form

$$
L=-\frac{1}{2 \kappa} L^{g}+L^{m}
$$

where $L^{g}$ is defined by Eq. (2.3) and $L^{m}$ is supposed to depend on $h^{\alpha \beta}, \varphi_{A}, \gamma^{\alpha \beta}, \stackrel{\varphi}{\varphi}_{A}$ and their first derivatives.

The characteristic feature of physical systems which include the background sources $\left(\stackrel{\circ}{\varphi}_{A}\right.$ in the present case) is the existence of "excitations" which contain coupled gravitational and matter variables on an equal footing. In other words, the variables $h^{\mu \nu}, \varphi_{A}$, if treated as infinitesimally small, satisfy the coupled linear equations and can be combined into a single multicomponent field. The distinction of different degrees of freedom and, in particular, the singling out of free gravitational waves is only possible at the sufficiently symmetric backgrounds [13]. (For a recent discussion of the criteria of gravitational radiation at the Friedmann-Robertson-Walker backgrounds, see [14].) 

form

The linear operator acting on $h^{\mu \nu}$, i.e. the 1.h.s. of Eq. (2.8) can be written in the

$$
G_{\mu \nu}^{L}(h) \equiv \frac{1}{\sqrt{-\gamma}} \frac{\delta}{\delta \gamma^{\mu \nu}}\left(\tilde{h}^{\alpha \beta} \frac{\delta \stackrel{L}{g}^{g}}{\delta \tilde{\gamma}^{\alpha \beta}}\right) .
$$

This operator takes its origin in the gravitational part, $\stackrel{\circ}{L}^{g}$, of the background action. In a similar fashion one can write the linear operator associated with $\check{L}^{m}$ :

$$
\Phi_{\mu \nu}^{L}(h, \varphi) \equiv-\frac{2 \kappa}{\sqrt{-\gamma}} \frac{\delta}{\delta \gamma^{\mu \nu}}\left[\tilde{h}^{\alpha \beta} \frac{\delta \dot{L}^{m}}{\delta \tilde{\gamma}^{\alpha \beta}}+\varphi_{B} \frac{\delta \dot{L}^{m}}{\delta \dot{\varphi}_{B}}\right] .
$$

The dynamical equations are to be derived from (3.3) by varying $L$ with respect to gravitational and matter variables respectively. First, consider the equations which result from varying $L$ with respect to gravitational variables (gravitational equations). The left hand side (linear part) of these equations in their second order form must consist of $G_{\mu \nu}^{L}+\Phi_{\mu \nu}^{L}$. Let us require that the right hand side of these equations be the total energy-momentum tensor following from (3.3). More precisely we require that the gravitational equations have the form

$$
G_{\mu \nu}^{L}+\Phi_{\mu \nu}^{L}=\kappa T_{\mu \nu}^{\mathrm{tot}} \equiv \kappa\left(t_{\mu \nu}+\frac{2}{\sqrt{-\gamma}} \frac{\delta L^{m}}{\delta \gamma^{\mu \nu}}\right) .
$$

From (3.3), by a computation similar to that described in Sect. 2, one gets

$$
G_{\mu \nu}^{L}=\kappa\left(t_{\mu \nu}+2 \frac{\delta L^{m}}{\delta \tilde{h}^{\mu \nu}}-\gamma_{\mu \nu} \gamma^{\alpha \beta} \frac{\delta L^{m}}{\delta \tilde{h}^{\alpha \beta}}\right) .
$$

Now, add Eq. (3.5) to the both sides of these equations and introduce the notation

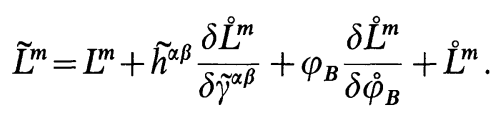

Then, Eq. (3.7) transforms into

$$
\begin{aligned}
G_{\mu \nu}^{L}+\Phi_{\mu \nu}^{L}= & \kappa\left[t_{\mu \nu}+2 \frac{\delta \tilde{L}^{m}}{\delta \tilde{h}^{\mu \nu}}-\gamma_{\mu \nu} \gamma^{\alpha \beta} \frac{\delta \tilde{L}^{m}}{\delta \tilde{h}^{\alpha \beta}}-\frac{2}{\sqrt{-\gamma}} \frac{\delta \dot{L}^{m}}{\delta \gamma^{\mu \nu}}\right. \\
& \left.-\frac{2}{\sqrt{-\gamma}} \frac{\delta}{\delta \gamma^{\mu \nu}}\left(\tilde{h}^{\alpha \beta} \frac{\delta \dot{L}^{m}}{\delta \gamma^{\alpha \beta}}+\varphi_{B} \frac{\delta \dot{L}^{m}}{\delta \dot{\varphi}_{B}}\right)\right] .
\end{aligned}
$$

In order to have $T_{\mu \nu}^{\text {tot }}$ at the r.h.s. of Eq. (3.8) it is sufficient [thanks to definitions (1.1) and (2.9)] to demand that $\tilde{h}^{\mu v}$ and $\tilde{\gamma}^{\mu v}$ appear as a sum in $\widetilde{L}^{m}$. It means that $\tilde{L}^{m}$ has to depend on the arguments: $\tilde{\gamma}^{\mu \nu}+\tilde{h}^{\mu \nu}, \varphi_{A}, \dot{\varphi}_{A}$ and their first derivatives. As a consequence,

$$
\frac{\delta \tilde{L}^{m}}{\delta \tilde{h}^{\mu \nu}}=\frac{\delta \tilde{L}^{m}}{\delta \tilde{\gamma}^{\mu \nu}}
$$

and the r.h.s. of (3.8) can be easily reduced to $\kappa T_{\mu v}^{\text {tot }}$. 
Now, consider the equations which result from varying $L$ with respect to matter variables (matter equations). The left hand side (linear part) of these equations must be

$$
\Phi_{A}^{L} \equiv-\frac{\delta}{\delta \dot{\varphi}_{A}}\left(\varphi_{B} \frac{\delta \stackrel{\circ}{m}^{m}}{\delta \dot{\varphi}_{B}}+\tilde{h}^{\alpha \beta} \frac{\delta \dot{L}^{m}}{\delta \tilde{\gamma}^{\alpha \beta}}\right),
$$

since this is precisely the linear term of the "perturbed" equations (3.2b). Let us require that the r.h.s. of matter equations be the "current" $J_{A}$ which is a result of varying $L^{m}$ with respect to $\stackrel{\varphi}{\varphi}_{A}$. In other words we want the equations to have the form:

$$
\Phi_{A}^{L}=J_{A} \equiv \frac{\delta L^{m}}{\delta \stackrel{\circ}{A}_{A}} .
$$

In order to satisfy this requirement it is sufficient to suppose that $\tilde{L}^{m}$ depends on $\varphi_{A}$ $+\stackrel{\circ}{\varphi}_{A}$. Thus, finally, we come to the conclusion that $L^{m}$ must be of the form:

$$
L^{m}=\tilde{L}^{m}-\tilde{h^{\alpha \beta}} \frac{\delta \check{L}^{m}}{\delta \tilde{\gamma}^{\alpha \beta}}-\varphi_{B} \frac{\delta \check{L}^{m}}{\delta \stackrel{\circ}{\varphi}_{B}}-\stackrel{\circ}{L}^{m}
$$

where $\tilde{L}^{m}$ depends on $\tilde{\gamma}^{\mu v}+\tilde{h}^{\mu v}, \varphi_{A}+\stackrel{\varphi}{\varphi}_{A}$ and their derivatives. If the terms $\stackrel{\circ}{ }^{m}$ and

$$
\tilde{h^{\alpha \beta}} \frac{\delta \dot{L}^{m}}{\delta \tilde{\gamma}^{\mu \nu}}+\varphi_{B} \frac{\delta \dot{L}^{m}}{\delta \dot{\varphi}_{B}}
$$

are, correspondingly, the zero order and the linear terms in the decomposition of $\widetilde{L}^{m}$ by powers of the dynamical variables $\widetilde{h}^{\mu v}, \varphi_{A}$ (with the total derivatives ignored), then $L^{m}$ contains not less than quadratic powers of the dynamical variables. Consequently, $T_{\mu \nu}^{\text {tot }}$ and $J_{A}$ also contain not less than quadratic dynamical terms.

After the identification (2.11) and $\stackrel{\circ}{A}_{A}+\varphi_{A} \equiv \tilde{\varphi}_{A}$ the dynamical equations (3.6), (3.10) [with (3.11) taken into account] together with the background equations (3.2) become equivalent to the Einstein equations and the matter field equations, written in terms of $g^{\mu \nu}$ and $\tilde{\varphi}_{A}$. The Lagrangian (3.3) can be viewed as the result of subtracting the zero-order and linear terms from the general Lagrangian

$$
-\frac{\sqrt{-g} R(g)}{2 \kappa}+\tilde{L}^{m}(g, \varphi)
$$

decomposed in powers of $\tilde{h}^{\mu v}$ and $\varphi_{A}$.

Since, in general, the covariant divergence of the 1.h.s. of (3.6) does not vanish, one has the relation $T_{\mu \nu}^{\mathrm{tot} ;} \neq 0$. The physical reason for this nonconservation is the interaction with the background system which exerts the "force" and "parametric" action on the dynamical fields. The mathematical origin of this relation will be discussed below (Sect. 4).

\section{3b) Background Curvature Without Background Matter}

In the preceding section it was assumed that the background Ricci tensor was governed by the background matter. However, one can imagine a situation where $\dot{T}_{\mu \nu}=0$, while $\dot{R}_{\mu \nu} \neq 0$. It means that $\dot{R}_{\mu \nu}$ is fixed "by hand" or is governed by some sources which we are, presently, not interested in. It is worth considering such a theory, yet it may run into inconsistency. 
One easily obtains a theory of this kind if considers the action (2.2) [or the action (2.13), (2.19) when dynamical matter is included] for such background metrics $\gamma^{\alpha \beta}$ that $R_{\mu \nu} \neq 0$. In this case the dynamical field equations maintain their form (2.4), (2.5) (or (2.14), (2.5)) though they may not be totally integrable now. In addition, the solutions of these field equations can be shown to satisfy the conservation law $t_{\mu \nu} ; v=0\left(\right.$ or $\left.T_{\mu \nu}^{\text {tot } ; v}=0\right)$ and the relation $G_{\mu \nu}^{L ; v}=0$, despite the fact that $G_{\mu \nu}^{L ; v} \neq 0$ due to (2.10) (for more detail about why this happens, see Sect. 4). However, the major defect of this theory is that it has no direct relation to GR. Indeed, the 1.h.s. of Eqs. (2.8) and (2.18) does not have the term

$$
\stackrel{\circ}{G}_{\mu \nu} \equiv \stackrel{\circ}{R}_{\mu \nu}-\frac{1}{2} \gamma_{\mu \nu} \stackrel{\circ}{R}
$$

while this term must be present in equations derived from Einstein's equations.

In order to remedy that defect and restore that term, one has to supply the Lagrangian $L^{g}$ with the additional contribution $L^{1} \equiv \widetilde{h}^{\mu v} R_{\mu \nu}^{\circ}$. Thus, we will consider the Lagrangian

$$
L=-\frac{1}{2 \kappa}\left(L^{g}+L^{1}\right)+L^{m}
$$

where $L^{m}$ is defined by (2.19). The varying of $L$ by $\tilde{h}^{\mu v}$ yields the equations

$$
\stackrel{\circ}{R}_{\mu \nu}+r_{\mu \nu}(K)=2 \kappa \frac{\delta L^{m}}{\delta \widetilde{h}^{\mu \nu}},
$$

and Eqs. (2.5), (2.15) remain unchanged. After the proper rearrangements one gets the second order field equations

$$
\stackrel{\circ}{G}_{\mu \nu}+G_{\mu \nu}^{L}=\kappa\left(t_{\mu \nu}+T_{\mu \nu}\right)
$$

where the expressions for $t_{\mu \nu}$ and $T_{\mu \nu}$ are determined by $-\frac{L^{g}}{2 \kappa}$ and $L^{m}$, as before. This seems to be a reasonable definition for the energy-momentum tensor since it implies that $T_{\mu \nu}^{\text {tot }} \equiv t_{\mu \nu}+T_{\mu \nu}$ is computed from that part of the total Lagrangian $L$ which is not less than quadratic in dynamical variables. In Eq. (3.13), $G_{\mu \nu}$ plays a role of the external "force," acting on the dynamical system. $T_{\mu \nu}^{\mathrm{tot}}$ is not conserved: $T_{\mu \nu}^{\mathrm{tot} ; v} \neq 0$.

If one computed $T_{\mu \nu}^{\text {tot }}$ from the total Lagrangian $L$, one would obtain

$$
T_{\mu \nu}^{\mathrm{tot}}(L) \equiv t_{\mu \nu}+T_{\mu \nu}-\frac{1}{\kappa} G_{\mu \nu}^{L}
$$

which is another way of saying that $G_{\mu \nu}^{L}$ is transferred from the 1.h.s. to the r.h.s. of Eq. (3.13). Such a definition of $T_{\mu \nu}^{\text {tot }}$ is hardly reasonable despite the fact that it gives $T_{\mu \nu}^{\mathrm{tot} ; v}=0$.

A further modification of the theory would be to consider the total Lagrangian $L=-\frac{1}{2 \kappa}\left(L^{0}+L^{1}+L^{g}\right)+L^{m}$, where $\stackrel{\circ}{L} \equiv \sqrt{-\gamma} \stackrel{R}{ }$. The field equations will again have the form of Eq. (3.13), but $T_{\mu \nu}^{\text {tot }}$ computed from this total Lagrangian would be given by

$$
T_{\mu \nu}^{\mathrm{tot}}=t_{\mu \nu}+T_{\mu \nu}-\frac{1}{\kappa}\left(\stackrel{\ominus}{G}_{\mu \nu}+G_{\mu \nu}^{L}\right),
$$


which is zero due to (3.13). A similar situation occurs in GR when one tries to use $g^{\mu \nu}$ both as the field variables and the metric components, which are used in the definition (1.1) of the energy momentum tensor. The origin of this disappointing result is now clear.

The field equations (3.13) do occur in current research [15]. In the quadratic approximation with respect to small $h^{\mu \nu}$ (and with $T_{\mu \nu}=0$ ) these equations were studied in [15a]. Under a special choice of the parameters of the small perturbations, the approximate solution to (3.13) can be interpreted as the propagation of gravitational waves $\left(G_{\mu \nu}^{L}=0\right)$ at the curved background space-time caused by the waves themselves $\left(\hat{G}_{\mu \nu}=\kappa t_{\mu \nu}\right)[15]$.

\section{The Gauge Invariance and the Conservation Laws}

A coordinate transformation can be regarded as a mapping of a space-time to itself. In this way the coordinate invariance of a physical system can be assigned the meaning of the gauge invariance. By the gauge invariance one usually understands the invariance with respect to some replacement of the background and dynamical variables in the same coordinate frame of reference. We will also introduce and use (at the end of Sect. 4) the notion of true gauge invariance which means the invariance with respect to replacement of the dynamical variables only. This property is fully analogous to the usual (internal) gauge invariance.

Let the mapping of the space-time be realized with the help of a vector field $\xi^{\alpha}\left(x^{\mu}\right)$. To a point, with the coordinates $x^{\alpha}$ one maps another point with coordinates $\tilde{x}^{\alpha}$. For a given mapping one can perform a coordinate transformation to the new coordinate system $x^{\alpha \prime}$ such that the mapped point $\tilde{x}^{\alpha}$ will have exactly the same numerical values of the new coordinates as the original point $x^{\alpha}$ has had in the old coordinate system.

By comparing the values of an object, say, a tensor field $\Omega_{\cdot \beta}^{\alpha}$, given in the original point and the old coordinate system with the values of the same object, $\Omega_{{ }_{\beta} \alpha^{\prime}}$, given in the mapped point and the new coordinate system, one arrives at the notion of the Lie-derivative [16]:

$$
L_{\xi} \Omega_{\cdot \beta}^{\alpha} \equiv \xi^{\varrho} \Omega_{\cdot \beta, \varrho}^{\alpha}+\xi_{, \beta}^{\varrho} \Omega_{\cdot \varrho}^{\alpha}-\xi_{, \varrho}^{\alpha} \Omega_{\beta}^{\varrho} .
$$

This expression keeps only the terms linear in $\xi^{\alpha}$. It can be shown that the exact relation, which includes all powers of $\xi^{\alpha}$, has the following form:

$$
\Omega_{\cdot{ }_{\beta}}^{\alpha^{\prime}}=\Omega_{\cdot \beta}^{\alpha}+\sum_{k=1}^{\infty} \frac{1}{k !} L_{\xi}^{k} \Omega_{\cdot \beta}^{\alpha},
$$

where $L_{\xi}^{k}$ means the Lie-derivative taken $k$ times. We are interested in the exact relation (4.1) since we will be interested in the full nonlinear theory.

Let us accept Eq. (4.1) as a gauge transformation for the background and dynamical variables. The concrete form of the gauge transformation depends on the coordinate properties of the transformed object.

Any Lagrangian, being a scalar density, acquires the total divergence under the transformation (4.1). For instance, in the approximation, linear in $\xi^{\alpha}$, Eq. (4.1) gives:

$$
L^{\prime}=L+\left(L \xi^{\alpha}\right)_{, \alpha}
$$


From (4.2) it is seen that the equations of motion will not be changed. If, additionally, one assumes that $\xi^{\alpha}$ vanishes at the boundary $\Sigma$ of the region of integration,

$$
\xi_{\mid \Sigma}^{\alpha}=0,
$$

then the action remains entirely unchanged - the property which may be important in the quantum version of the theory. (The interrelation between coordinate and gauge symmetries and their role in quantum gravity is discussed in [17].)

The direct use of Eq. (4.1) in the equations of motion transform them into a combination of the same equations. For instance, if one puts the expressions for $K_{\mu \nu}^{\alpha \prime}, \gamma^{\mu v^{\prime}}$ into Eq. (2.4) then the 1.h.s. of these equations transforms into $r_{\mu \nu}^{\prime}$, where

$$
r_{\mu \nu}^{\prime}=r_{\mu \nu}+\sum_{k} \frac{1}{k !} L_{\xi}^{k} r_{\mu \nu} .
$$

Thus, the replacement (4.1) with the arbitrary $\xi^{\alpha}(x)$ transfers any solution of the field equations into another solution of the same equations. In this sense the equations of motion are gauge invariant. The energy-momentum tensor $t_{\mu \nu}$ by itself is not gauge invariant with respect to (4.1). However, it is not invariant exactly to the same extent that the quantity $G_{\mu \nu}^{L}$ is not invariant, for which $t_{\mu \nu}$ is the source term.

The gauge symmetries of the action are closely connected with the conservation laws for $T_{\mu \nu}^{\text {tot }}$ which we derived in Sect. 2 and Sect. 3 by direct computations from the equations of motion. We will show this in detail. For simplicity, we restrict ourselves to the vector field $\xi^{\alpha}(x)$ which satisfies Eq. (4.3) and, additionally, the restrictions:

$$
\xi_{, \beta \mid \Sigma}^{\alpha}=0, \quad \xi_{, \beta, \gamma \mid \Sigma}^{\alpha}=0 .
$$

These conditions allow us to ignore the total divergences under the sign of the integral. However, the restrictions (4.3), (4.4) are not compulsory and the conclusions formulated below could be obtained without use of (4.3), (4.4), though technically more difficult. The conclusions about $T_{\mu v}^{\text {tot; } v}$ could be also derived as a result of application of Noether's theorems (see, for example [18, 19]).

For our purposes it is sufficient to take account of terms linear in $\xi^{\alpha}$ only. Because of Eqs. (4.2), (4.3) the action is gauge invariant, i.e. its increment vanishes. It is necessary to write down this fact in more detail. For this, one has to take into account the concrete dependence of $L$ on its arguments and use $L_{\xi} \Omega^{\alpha}{ }_{\beta}$ as their increments. Further, one has to use the fact that the ordinary and Lie-derivatives commute, and integrate by parts with the use of Eqs. (4.3), (4.4). Then, for the action (2.2), one gets

$$
\int d^{4} x\left\{\frac{\delta L}{\delta \tilde{h}^{\mu \nu}} L_{\xi} \tilde{h}^{\mu \nu}+\frac{\delta L}{\delta K^{\alpha}{ }_{\mu \nu}} L_{\xi} K^{\alpha}{ }_{\mu \nu}+\frac{\delta L}{\delta \gamma^{\mu \nu}} L_{\xi} \gamma^{\mu \nu}\right\} \equiv 0 .
$$

If the equations of motion are satisfied, the first two terms vanish. Since

$$
L_{\xi} \gamma^{\mu v}=-\xi^{\mu ; v}-\xi^{v ; \mu},
$$


the integration by parts of the last term in (4.5) leads to the final conclusion: $t^{\mu v} ; v$ $=0$. It should be noted that Eq. (2.1) has not been used in the course of derivation, so that this conservation law is valid even if $\dot{R}_{\mu \nu} \neq 0$. This fact was mentioned in Sect. $3 b$.

In the case when matter is present (Sect. 2b), Eq. (4.5) has the additional term $\frac{\delta L}{\delta \varphi_{A}} L_{\xi} \varphi_{A}$. This term vanishes if the matter field equations (2.15) are satisfed. As a result, one comes to the conservation law (2.20).

The relation $T_{\mu v}^{\text {tot } ;} \neq 0$ revealed in Sect. 3a can also be easily explained. In this case one obtains:

$\int d^{4} x\left\{\frac{\delta L}{\delta \tilde{h}^{\mu \nu}} L_{\xi} \tilde{h}^{\mu \nu}+\frac{\delta L}{\delta K^{\alpha}{ }_{\mu \nu}} L_{\xi} K^{\alpha}{ }_{\mu \nu}+\frac{\delta L}{\delta \varphi_{A}} L_{\xi} \varphi_{A}+\frac{\delta L}{\delta \gamma^{\mu \nu}} L_{\xi} \gamma^{\mu \nu}+\frac{\delta L}{\delta \stackrel{\circ}{A}_{A}} L_{\xi} \stackrel{\circ}{\varphi}_{A}\right\} \equiv 0$,

instead of Eq. (4.5). The first three terms disappear because of the equations of motion. However, the last term does not vanish and, consequently, $T_{\mu \nu}^{\text {tot; } v} \neq 0$. Again, the explicit relation, which follows from Eq. (4.7), is, after all, a consequence of the field equations. In order to derive this relation one has to rewrite the last term of Eq. (4.7) in such a way that it is proportional to $L_{\xi} \gamma^{\mu \nu}$. Then, by using Eqs. (3.9), (3.10), and Eq. (3.2) along with the Lie-derivatives of Eq. (3.2), the last two terms in (4.7) can be combined and rearranged in such a way that after integrating by parts, Eq. (4.7) yields:

$$
\left(G_{\mu \nu}^{L}+\Phi_{\mu \nu}^{L}-\kappa T_{\mu \nu}^{\mathrm{tot}}\right)^{; \nu}=0 .
$$

This relation is trivially satisfied because of Eq. (3.6).

Up to this moment we have been considering the background and dynamical variables on the same footing. Both classes of variables underwent the gauge transformations (4.1). Now we will show that the theory is also invariant with respect to the so-called true gauge transformations, i.e. the transformations applied to the dynamical variables only. As the true gauge transformations we accept the following:

$$
\begin{aligned}
\tilde{h}^{\alpha \beta \prime} & =\tilde{h}^{\alpha \beta}+\sum_{k} \frac{1}{k !} L_{\xi}^{k}\left(\tilde{h}^{\alpha \beta}+\tilde{\gamma}^{\alpha \beta}\right), \\
K_{\mu \nu}^{\alpha \prime} & =K_{\mu \nu}^{\alpha}+\sum_{k} \frac{1}{k !} L_{\xi}^{k}\left(K_{\mu \nu}^{\alpha}+C_{\mu \nu}^{\alpha}\right), \\
\varphi_{A}^{\prime} & =\varphi_{A}+\sum_{k} \frac{1}{k !} L_{\xi}^{k}\left(\varphi_{A}+\stackrel{\circ}{A}_{A}\right) .
\end{aligned}
$$

Unlike Eq. (4.1) these transformations are inhomogeneous since they contain the background quantities.

With respect to the transformations (4.9) an action is only invariant if the background equations of motion are fulfilled. For instance, by inserting $\tilde{h}^{\alpha \beta \prime}, K_{\mu \nu}^{\alpha \prime}$ into (2.3) one transforms $L^{g}$ in the following way:

$$
L^{g^{\prime}}=L^{g}-\stackrel{R}{\alpha \beta}_{k} \sum_{k} \frac{1}{k !} L_{\xi}^{k}\left(\tilde{h}^{\alpha \beta}+\tilde{\gamma}^{\alpha \beta}\right)+\operatorname{div},
$$


where div denotes the total divergences irrelevant owing to Eqs. (4.3), (4.4). Thus, the action (2.2) is only invariant with respect to transformations (4.9) if Eqs. (2.1) are satisfied [as well as Eqs. (4.3), (4.4)]. Under the same conditions the action (2.13) is invariant. The action based on Eq. (3.3) is invariant if Eqs. (3.2), (4.3), (4.4), are fulfilled.

Under the transformations (4.9) the dynamical field equations transform into a combination of themselves if the background field equations are fulfilled. For instance, Eq. (2.8) is invariant if $R_{\mu \nu}=0$. In other words, if $\hat{R}_{\mu \nu}=0$ and the given set of $\widetilde{h}^{\mu \nu}, K_{\mu \nu}^{\alpha}$ is a solution to Eq. (2.8) [or, to be more precise, is a solution to Eqs. (2.4), (2.5)], then $\tilde{h}^{\mu \nu \prime}, K_{\mu \nu}^{\alpha \prime}$ will also be a solution.

The energy-momentum tensor $t_{\mu \nu}$ by itself is not invariant with respect to the gauge transformations (4.9) and acquires the additional term in the form of a covariant divergence. The substitution of $\tilde{h}^{\alpha \beta \prime}$ and $K_{\mu \nu}^{\alpha \prime}$ into (2.9) transforms $t_{\mu \nu}$, with the help of Eqs. (2.4), (2.5), into

$$
t_{\mu \nu}^{\prime}=t_{\mu \nu}+\frac{1}{\kappa} G_{\mu \nu}^{L}\left[\frac{1}{\sqrt{-\gamma}} \sum_{k !} \frac{1}{k !} L_{\xi}^{k}\left(\tilde{h}^{\alpha \beta}+\tilde{\gamma}^{\alpha \beta}\right)\right],
$$

where the square brackets indicate the argument of the operator $G_{\mu v}^{L}$. Since the operator $G_{\mu \nu}^{L}$, according to its definition, is a covariant divergence

$$
2 G_{\mu v}^{L}(h) \equiv\left(h_{\mu \nu}{ }^{; \alpha}+\gamma_{\mu \nu} h_{; \beta}^{\beta \alpha}-h_{v ; \mu}^{\alpha}-h_{\mu ; \nu}^{\alpha}\right)_{; \alpha},
$$

the additional term in (4.11) is also a covariant divergence. Equation (4.11) expresses the fact of the gauge invariance of Eq. (2.8) with respect to (4.9). Obviously, one maintains $t_{\mu \nu}^{\prime} ; \nu=0$.

Equation (4.9) imply the existence of the "pure gauge" solutions, i.e. such solutions $\widetilde{h}^{\alpha \beta^{\prime}} \neq 0, K_{\mu \nu}^{\alpha \prime} \neq 0$ which are generated from $\widetilde{h}^{\alpha \beta}=0, K_{\mu \nu}^{\alpha}=0$ by the use of Eqs. (4.9). The origin of these solutions can be also explained in the following way. One could apply a coordinate transformation to the background metric density $\tilde{\gamma}^{\mu \nu}\left(x^{\alpha}\right)\left(R_{\mu \nu}=0\right)$ and decompose the result into the sum $\tilde{\gamma}^{\mu v}\left(\tilde{x}^{\alpha}\right)+\tilde{h}^{\mu v}\left(\tilde{x}^{\alpha}\right)$, where $\tilde{\gamma}^{\mu \nu}\left(\tilde{x}^{\alpha}\right)$ are the functions of the new coordinates exactly the same as $\tilde{\gamma}^{\mu \nu}\left(x^{\alpha}\right)$. The rest, $\tilde{h}^{\mu \nu}\left(\tilde{x}^{\alpha}\right)$, are the "pure gauge" solutions. It follows from (4.11) that $t_{\mu v}$ constructed from these solutions can be transformed away by the gauge transformation.

As usual, one can connect the invariance of the action with some identities similar to the ones that lead to the current conservation in electrodynamics and the Yang-Mills fields. In the present case one deals with Eq. (4.9) together with the background equations of motion. As a result of this symmetry one obtains Eqs. (2.20), (4.8) which are already known.

Let us turn to the integral conservation laws. From a covariant differential law (2.20) it follows that there exist the coordinate independent conserved quantities if the background space-time is symmetric, i.e. admits the group of motions. It is well known that in the flat space-time the use of the curvilinear coordinates does not prevent obtaining the integral conservation laws. On the other hand, the always possible rewriting of the covariant differential law in the form of an ordinary divergence does not help to obtain the coordinate independent integral quantities if the space-time does not admit motions. Since GR can be always formulated at the flat background (Sect. 2b) the number of the integral conserved quantities (for a 
system with appropriate conditions at infinity) is equal to 10 - the number of the Killing vectors. Recall that $t_{\mu \nu}$ is a tensor, so that a choice of the coordinate system is absolutely indifferent. On the contrary, the usual approach gives the meaningful results, even in an asymptotically flat space-time, if only the asymptotically Minkowskian coordinates are used [3].

\section{Properties of the Energy-Momentum Tensor and Some Examples}

Let us consider some properties of $t_{\mu \nu}$ additional to those already mentioned in Sect. 2. Because of Eq. (2.4) $t_{\mu \nu}$ can be represented as a covariant divergence:

$$
t_{\mu \nu}=\frac{1}{\kappa}\left[K_{\mu \nu}^{\alpha}-\frac{1}{2} \delta_{\mu}^{\alpha} K_{v}-\frac{1}{2} \delta_{v}^{\alpha} K_{\mu}-\frac{1}{2} \gamma_{\mu v}\left(\gamma^{\tau \varrho} K_{\tau \varrho}^{\alpha}-\gamma^{\alpha \varrho} K_{\varrho}\right)+Q_{\mu \nu}^{\alpha}\right]_{; \alpha} .
$$

The further use of Eq. (2.5) leads to Eq. (2.8), i.e. $t_{\mu v}$, due to Eqs. (2.4), (2.5), is a double divergence:

$$
t_{\mu \nu}=\frac{1}{2 \kappa}\left[h_{\mu \nu} \gamma^{\alpha \beta}+h^{\alpha \beta} \gamma_{\mu \nu}-h_{\mu}^{\beta} \delta_{\nu}^{\alpha}-h_{v}^{\beta} \delta_{\mu}^{\alpha}\right]_{; \alpha ; \beta} .
$$

In the presence of matter the similar formula is valid for $t_{\mu \nu}+T_{\mu \nu}$ (Sect. 2b).

Expressions, like Eqs. (5.1), (5.2), permit one, as usual, to reduce the volume integrals to the surface ones. As a result, the integral characteristics of an isolated system can be expressed in terms of the asymptotic values of the fields.

Let the background be flat, then one can choose $\gamma^{\mu \nu}=\eta^{\mu \nu}, C_{\mu \nu}^{\alpha}=0$. Define an isolated system by the conditions:

$$
\begin{aligned}
h^{\mu \nu} & =O\left(\frac{1}{r}\right), & h_{, \alpha}^{\mu \nu}=O\left(\frac{1}{r^{2}}\right), \\
K_{\mu \nu}^{\alpha} & =O\left(\frac{1}{r^{2}}\right), & K_{\mu \nu, \beta}^{\alpha}=O\left(\frac{1}{r^{3}}\right) .
\end{aligned}
$$

Such a definition agrees with what is known as an asymptotically flat space-time in $\mathrm{GR}$, i.e. with the definition:

$$
\begin{gathered}
g_{\mu \nu}=\eta_{\mu \nu}+O\left(\frac{1}{r}\right), \quad g_{\mu v, \alpha}=O\left(\frac{1}{r^{2}}\right), \\
\Gamma_{\mu \nu}^{\alpha}=O\left(\frac{1}{r^{2}}\right), \quad \Gamma_{\mu \nu, \beta}^{\alpha}=O\left(\frac{1}{r^{3}}\right) .
\end{gathered}
$$

For the isolated system, defined by Eq. (5.3), one has $L^{g}=O\left(1 / r^{4}\right), t_{\mu \nu}=O\left(1 / r^{4}\right)$, and the action is finite for such a system. Since the action is a scalar and $t_{\mu \nu}$ is a tensor, a specific choice of the frame of reference is of no importance. In particular, the use of the spherical coordinates instead of the Cartesian coordinates is entirely harmless, which is quite different from the usual approach based on pseudotensors. Any spatial transformations can not change the integral value for the energy

$$
P^{0}=\int t^{00} \sqrt{-\gamma^{(3)}} d^{3} x .
$$


It has to be mentioned that the "pure gauge" solutions generated by (4.9), with the vector field $\xi^{\alpha}\left(x^{\mu}\right)$ restricted by (4.3), (4.4), possess zero total energy.

It may be interesting to establish a link between $t^{\mu v}$ and a pseudotensor, say, the Landau-Lifshitz pseudotensor $t_{L L}^{\mu \nu}$ [2]. Inserting $\sqrt{-g} g^{\mu \nu}=\eta^{\mu v}+\tilde{h}^{\mu \nu}$ into $t_{L L}^{\mu \nu}$ and using the Minkowskian coordinates one can show that

$$
t^{\mu v}=-g t_{L L}^{\mu \nu}-\frac{1}{2 \kappa}\left(h^{\mu \nu} h^{\alpha \beta}-h^{\alpha \mu} h^{\beta v}\right)_{, \alpha, \beta} .
$$

Exactly this quantity shows up in the equations of motion of isolated bodies with the radiation reaction force taken into account (see, for example [20]).

As examples of the application of $t_{\mu \nu}$, consider the Schwarzschild solution and the weak gravitational waves. First, consider the Schwarzschild solution in its usual form:

$$
d s^{2}=\left(1-\frac{r_{g}}{r}\right) c^{2} d t^{2}-\frac{d r^{2}}{1-\frac{r_{g}}{r}}-r^{2}\left(d \theta^{2}+\sin ^{2} \theta d \varphi 2\right) ; \quad r_{g}=\frac{2 G m}{c^{2}} .
$$

It is natural to treat (5.5) as the field:

$$
h^{00}=\frac{r_{g}}{r-r_{g}} ; \quad h^{11}=\frac{r_{g}}{r},
$$

given on the flat background space-time written in the spherical coordinates:

$$
\begin{gathered}
\gamma^{00}=1, \quad \gamma^{11}=-1, \quad \gamma^{22}=-\frac{1}{r^{2}}, \\
\gamma^{33}=-\frac{1}{r^{2} \sin ^{2} \theta} ; \quad \sqrt{-\gamma^{(3)}}=r^{2} \sin \theta .
\end{gathered}
$$

The expression (5.4) for $P^{0}$ rearranged with the help of Eq. (5.2) gives

$$
\begin{aligned}
P^{0} & =\frac{1}{2 \kappa} \int\left(\frac{r_{g}}{r-r_{g}} \gamma^{j k}+h^{j k}\right)_{|k| j} \sqrt{-\gamma^{(3)}} d^{3} x \\
& =\frac{1}{2 \kappa} \int\left[\left(\frac{r_{g}}{r-r_{g}} \gamma^{j k}+h^{j k}\right)_{\mid k} \sqrt{-\gamma^{(3)}}\right]_{, j} d^{3} x \\
& =\frac{1}{2 \kappa} \lim _{r \rightarrow \infty} \oint\left(\frac{r_{g}}{r-r_{g}} \gamma^{j k}+h^{j k}\right)_{\mid k} \sqrt{-\gamma^{(3)}} d S_{j},
\end{aligned}
$$

where the bar means the covariant derivatives with respect to the 3-metric $\gamma^{11}, \gamma^{22}$, $\gamma^{33}$. The calculation in (5.7) yields $P^{0}=m c^{2}$.

The Schwarzschild solution in the isotropic coordinates is

$$
d s^{2}=\frac{\left(1-r_{g} / 4 \varrho\right)^{2}}{\left(1+r_{g} / 4 \varrho\right)^{2}} c^{2} d t^{2}-\left(1+r_{g} / 4 \varrho\right)^{4}\left(d x^{2}+d y^{2}+d z^{2}\right) ; \quad r=\varrho\left(1+r_{g} / 4 \varrho\right)^{2} \text {. }
$$

This is easily treated as the gravitational field

$$
h^{00}=\frac{\left(1+r_{g} / 4 \varrho\right)^{7}}{1-r_{g} / 4 \varrho}-1 ; \quad h^{11}=h^{22}=h^{33}=\left(r_{g} / 4 \varrho\right)^{2},
$$


given on the flat background space-time written in the Minkowskian coordinates, $\gamma^{\mu \nu}=\eta^{\mu \nu}$. The total energy is

$$
P^{0}=\frac{1}{2 \kappa} \lim _{r \rightarrow \infty} \oint\left[\frac{2 r_{g}}{r^{2}} \frac{x^{j}}{r}+O\left(\frac{1}{r^{3}}\right)\right] d S_{j},
$$

which gives $P^{0}=m c^{2}$ again.

The solution for the weak gravitational waves in TT-gauge [3] is

$$
\begin{gathered}
d s^{2}=d x^{02}-d x^{2}-(1+a) d y^{2}-(1-a) d z^{2}-2 b d y d z ; \\
a=a\left(x^{0}-x\right), \quad b=b\left(x^{0}-x\right) .
\end{gathered}
$$

We can consider this metric as the gravitational field

$$
h^{22}=-h^{33}=a ; \quad h^{23}=b
$$

given on flat space-time, $\gamma^{\mu \nu}=\eta^{\mu \nu}$.

By using (5.8) one can compute $K_{\mu \nu}^{\alpha}$ according to Eq. (2.5) and then calculate $t_{\mu v}$. In the first nonvanishing approximation the following components of $t_{\mu \nu}$ are different from zero:

$$
t_{00}=\frac{2 c^{4}}{32 \pi G}\left[\left(a_{, 0}\right)^{2}+\left(b_{, 0}\right)^{2}\right] ; \quad t^{01}=t^{11}=t_{00} .
$$

This expression coincides with that derived from $t_{L L}^{\mu \nu}$.

Acknowledgements. One of us (L.G.) is indebted to S. Deser for a very valuable conversation.

\section{References}

1. Fock, V.A.: Theory of space, time, and gravitation. London: Pergamon 1959

2. Landau, L.D., Lifshitz, E.M.: The classical theory of fields. 3rd rev. English ed. Reading, MA. London: Addison-Wesley and Pergamon 1971

3. Misner, C.W., Thorne, K.S., Wheeler, J.A.: Gravitation. San Francisco: Freeman 1973

4. De Witt, B.S.: Dynamical theory of groups and fields. New York: Gordon and Breach 1965

5. Kraichnan, R.H.: Special-relativistic derivation of generally covariant gravitation theory. Phys. Rev. 98, 1118-1122 (1955)

6. Weinberg, S.: Photons and gravitons in perturbation theory: derivations of Maxwell and Einstein equations. Phys. Rev. B 138, 988-1002 (1965)

7. Ogiyevetsky, V.I., Polubarinov, I.V.: Interaction field of spin two and the Einstein equations. Ann. Phys. 35, 167-208 (1965)

8. Deser, S.: Self-interaction and gauge invariance. G.R.G. 1, 9-18 (1970)

9. Rosen, N.: Flat-space metric in general relativity theory. Ann. Phys. 22, 1-10 (1963)

10. Thorne, K.S., Lee, D.L., Lightman, A.P.: Foundations for a theory of gravitation theories. Phys. Rev. D 7, 3563-3578 (1973)

11. Deser, S., Laurent, B.E.: Gravitation without self-interaction. Ann. Phys. 50, 76-101 (1968)

12. Grishchuk, L.P., Zeldovich, Ya.B.: Complete cosmological theories. In:Quantum structure of space and time. Duff. M.J., Isham, C.J. (eds.). Cambridge: Cambridge University Press 1982

13. Grishchuk, L.P., Popova, A.D.: a) Gauge conditions for fields of higher spins in an external gravitational field. Sov. Phys. J.E.T.P. 53, 1-8 (1981); b) Space-times admitting the complete set of the gauge conditions for higher spin fields. J. Phys. A: Math. Gen. 15, 3525-3530 (1982) 
14. Niedra, J.M., Janis, A.I.: Gravitational radiation in Robertson-Walker backgrounds. G.R.G. 15, 241-254 (1983)

15. a) Isaacson, R.A.: Gravitational radiation in the limit of high frequency. Phys. Rev. 166, $1263-$ 1280 (1968)

b) Barnebey, T.A.: Gravitational waves: the nonlinearized theory. Phys. Rev. D 10, 1741-1748 (1974)

16. a) Schouten, J.A.: Tensor analysis for physicists. Oxford: Clarendon Press 1951

b) Eisenhart, L.P.: Continuous groups of transformations. Princeton, NJ: Princeton University Press 1933

17. Teitelboim, C.: Quantum mechanics of the gravitational field. Phys. Rev. D 25, 3159-3179 (1982)

18. a) Mitzkevich, N.V.: Physical fields in general relativity theory. Moscow: Nauka 1969 (in Russian)

b) Konopliova, N.P., Popov, V.N.: Gauge fields. Moscow: Atomizdat 1980 (in Russian)

19. Davis, W.R.: Classical fields, particles and the theory of relativity. New York: Gordon and Breach 1970

20. Grishchuk, L.P., Kopeykin, S.M.: On the motion of gravitating bodies with the radiation reaction force taken into account. Sov. Astron.: Pisma Astronom. J. 9, 436-440 (1983)

Communicated by Ya. G. Sinai

Received February 10, 1984 